\title{
Breast cancer surgery: when less is more
}

\author{
A Mannell \\ Department of Surgery, University of the Witwatersrand \\ Corresponding author: Aylwyn Mannell (mannell@mweb.co.za)
}

\begin{abstract}
Summary
This brief review traces the evolution of breast cancer surgery from radical mastectomy (MTX) to breast conserving therapy (BCT) where wide local excision (WLE) of the cancer is combined with whole breast irradiation. The role of sentinel lymph node biopsy (SLNB) to assess the axilla as an alternative to more extensive axillary lymph node dissection (ALND) is examined. This review is a contemporary evaluation of these procedures (BCT, SLNB) and their impact on survival and in reduction of morbidity, and shows that local recurrence of breast cancer (BC) after BCT has been reduced to $<5 \%$ at 10 year follow-up when guidelines that excision margins should be free of microscopic cancer are followed and where patients receive whole breast irradiation together with systemic therapy after WLE. Over the past decade, studies of women with early BC entered on the SEER data base, the California Cancer Register and the Norwegian Cancer Registry have shown that overall survival (OS) and disease specific survival (DSS) are significantly better after BCT than following MTX. The improved survival after BCT compared to MTX cannot be attributed to radiation alone. The critical difference between these two procedures is the extent of dissection. Experimental studies using animal models have provided convincing evidences that the more extensive the tissue trauma, the greater the postoperative inflammatory cascade. This cascade of cytokines and growth factors impairs humoral immunity and enhances cancer cell survival and growth. The reduction in tissue trauma in patients undergoing BCT is a likely explanation for their improved survival. Compared to ALND, SLNB results in less lymphoedema, sensory loss and impaired shoulder function. For the clinically negative axilla, normal on palpation and imaging, SLNB is accurate in both the initial assessment and after neoadjuvant chemotherapy (NAC).
\end{abstract}

S Afr J Surg 2019;57(1)

http://dx.doi.org/10.17159/2078-5151/2018/v57n1a2782

\section{Introduction}

This brief review traces the evolution of breast cancer surgery from total mastectomy to wide local excision with whole breast irradiation. Guidelines established to reduce loco-regional recurrence and to improve overall survival are identified.

The impact of sentinel lymph node biopsy (SLNB) to assess the axilla and reduce the morbidity of axillary lymph node dissection (ALND) is highlighted. The use of SLNB for the clinically negative axilla in both early breast cancer and following neoadjuvant chemotherapy for advanced disease is discussed.

\section{The breast}

For decades, even centuries, the only surgical option for women with breast cancer was mastectomy. In 1898, William
J Halsted described the results of 'Radical Mastectomy' (MTX) in which the breast, chest wall muscles, axillary contents and often supraclavicular as well as mediastinal nodes were excised. ${ }^{1}$ These attempts to cure breast cancer by excising increasing amounts of tissue in the mistaken belief that cancer spreads solely in a centrifugal fashion resulted in gross deformity, impaired upper limb function and patient despair with no significant improvement in survival. ${ }^{2}$ However, in the same year that Halsted's paper was published, a Polish scientist (Marie Curie née Sktdowska) discovered radium, performing experiments showing that radiation halted cell division and could effect local destruction of tissue. ${ }^{3}$ As a result, by the $1920 \mathrm{~s}$, clinicians began to use radiation in the treatment of breast cancer.

Geoffrey Keynes published a report of 50 patients with inoperable breast cancer treated by interstitial radium needles and described the disappearance of tumour with prolonged survival in $10 \%$ of these cases. ${ }^{4}$ By 1937 , Keynes had extended 
the use of radium for Stage I and II breast cancers reporting 5-year survivals of $71 \%$ and $31 \%$ respectively: note was made of the 'aesthetic advantages of conservative management'. ${ }^{5}$ In a non-randomised study at the Royal Infirmary Edinburgh, McWhirter ${ }^{6}$ compared the outcomes of over 1000 patients managed by radiation and either simple mastectomy or radical mastectomy. Five-year survival after the lesser procedure (62.1\%) compared favourably to that after radical mastectomy (50.1\%). Vera Peters at the Radiotherapy Institute, Toronto General Hospital, was able to offer radiotherapy to patients with breast cancer undergoing a diagnostic excision biopsy but who were either unfit for or refused mastectomy: the survival of early stage cases treated by local excision and radiation were as good as that achieved by radical mastectomy and with less disfigurement. ${ }^{7}$

Shortly after publication of her results in 1967, 6 prospective randomised controlled trials were begun in Italy, North America and Europe ${ }^{8,9}$ to compare survival after breast conserving surgery with radiation (BCT) to that after mastectomy. Survival equivalence was documented at 5 , 10, 15- and 20-year follow-ups. In June 1990, the National Institutes of Health (NIH) in America held a consensus conference on treatment of patients with early stage invasive breast cancer: a consensus statement recommended BCT for the majority of Stage I and II breast cancer. ${ }^{10}$

Despite the obvious advantages of $\mathrm{BCT}$ in retaining normal appearance, sensation, movement and with less effect on psychosexual function, mastectomy is frequently offered to patients with early breast cancer. Multiple factors contribute to this trend: risk of recurrence, availability of plastic reconstruction, unaffordability of, or lack of access to radiotherapy, the often unacceptable cosmetic results of BCT as well as fear of repeated operations. In the EORTC* 10801 study ${ }^{11}$ comparing BCT with mastectomy, $48 \%$ of patients had microscopically involved margins so that the 10-year local recurrence rate was an alarming $20 \%$ despite adjuvant therapy and radiation boost to the tumour bed. The importance of clear margins ('no tumour on the ink') was then confirmed by the meta-analysis performed by Houssani ${ }^{13}$ on over 28 000 patients after BCT: when the excision margin is $1 \mathrm{~mm}$ from microscopic tumour, local recurrence is less than $5 \%$ in those patients having $\mathrm{BCT}$ who receive adequate radiation and appropriate systemic treatment. Since it is no longer necessary to excise large amounts of normal breast tissue to obtain 'safe' margins, patients can be assured of cosmetically acceptable results. The use of intraoperative techniques such as frozen section, scrape cytology, specimen radiology and margin probes to confirm microscopic clearance help to dispel the fear of re-operations. ${ }^{14}$ Despite the reduced risk of local recurrence and repeated operations after BCT as well as improvements in adjuvant therapy radiation techniques and targeted treatments, mastectomy is often identified as "the safer option". Thus, the uptake of mastectomy by women with early BC from well-resourced areas has continued. ${ }^{15}$ This prompted a contemporary assessment of overall survival (OS) and disease specific survival (DSS) after mastectomy (MTX) compared to BCT. Hwang ${ }^{16}$ studied the outcomes of 112000 women with Stage I or II in the California Cancer Register between 1990 and 2004 and who were eligible for BCT. ${ }^{15}$

The finding that women undergoing MTX had a significantly lower OS and DSS compared to those who had BCT was unexpected. This finding was independent of age and hormonal status and was confirmed by a larger study of 130000 women with early $\mathrm{BC}$ on the SEER** data base. ${ }^{17}$ In the latter study, multivariate analysis showed that women having BCT had longer OS than those who had MTX or MTX with radiation, results which were independent of patient demographics and tumour biology. ${ }^{17}$ The improved survival after BCT in these contemporary American Series prompted two registry-based studies of Norwegian women with early BC for whom BCT or MTX were considered equally beneficial. In the 2015 report, ${ }^{18}$ the 5 -year DSS for women after primary BCT was $97 \%$ compared to $88 \%$ after primary MTX. In a later report, ${ }^{19}$ the 10 -year DSS for $\mathrm{T} 1 \mathrm{~N} 0^{20} \mathrm{BC}$ was similar but the 10-year DSS for T1N1 was $97 \%$ for women having BCT and $89 \%$ for women who had an MTX.

The limitations of retrospective registry reviews are well known; non-randomization and physician bias being among the confounding variables but the strength of these comparative studies of survival lies in the large amount of clinicopathological data collected, the long-term (ten year) follow-up and careful statistical analysis.

That the addition of radiation to MTX did not increase survival over MTX alone suggests that the improved survival following BCT is not a direct result of radiation to the breast. The critical difference between the two procedures is the extent of dissection and the amount of traumatised tissue.

The local and systemic inflammatory response to tissue trauma excites a cascade of cytokines, prostaglandins and catecholamines which promote the adherence and growth of circulating cancer cells. The more extensive the trauma (or dissection) the greater the inflammatory response and the impairment of humoral immunity. ${ }^{21}$ This has been demonstrated in both experimental models and in clinical surgery.

Da Costa $^{22}$ used a murine tumour model to show that the growth and number of pulmonary metastases was enhanced by laparotomy compared to laparoscopy. Measuring helper T-cell function as a marker of humoral immunity, Berguer ${ }^{23}$ studied patients undergoing vascular procedures: the suppression of the immune response after aortic surgery was significantly greater than after the lesser procedure of carotid endarterectomy.

When the guidelines to select women eligible for BCT are followed, and cases of multi quadrant cancers, diffuse malignant microcalcifications, previous therapeutic DXT, persistently positive resection margins and cancers in the first two trimesters of pregnancy are excluded, ${ }^{24}$ the lesser procedure of BCT can be safely recommended for early BC. There is now growing evidence that BCT for stage I and II disease has a significantly better DSS and a lower risk of dying from $\mathrm{BC}$ compared to women undergoing the more extensive surgical dissection required for MTX. ${ }^{19}$

*European Organization for Research and Treatment of 
Cancer

**Surveillance, Epidemiology and End Results

\section{The axilla}

For BC management, axillary lymph node dissection (ALND) has been a standard surgical procedure for prognostic assessment and to excise lymph node metastases. But when nodes are histologically normal, excision and destruction of axillary lymphatics is of no oncological benefit to the patient.

In 1991 Morton devised the sentinel lymph node biopsy (SLNB) technique, ${ }^{25}$ the sentinel nodes being the first to receive lymph drainage from a tumour and which can be identified by lymphoscintigraphy and/or Patent Blue Dye. ${ }^{26}$ With these techniques the SLNs are consistently found in over $95 \%$ of axillae and will accurately predict the status of the remaining axillary nodes ${ }^{26}$ False negative results vary from 5 to $8 \%$ using the combination of techniques: these findings were confirmed by a meta-analysis of 69 trials of SLNB in early breast cancer. ${ }^{26}$ SLNB has replaced the untargeted practice of "axillary sampling" which has a false negative rate of $24 \%{ }^{27}$ and has now become the 'gold standard' method of assessment of the clinically negative axilla (cN0), defined as an axilla that is normal both to palpation and imaging. Given the accuracy of axillary assessment and reduction in the morbidity associated with ALND, namely lymphoedema, sensory loss and impaired shoulder function, ${ }^{28}$ there has been global acceptance of this technique.

As targeted therapy such as antioestrogen medication is increasingly available to women with $\mathrm{BC}$, it is possible to avoid even SLNB in those in whom the results would not alter treatment. These include elderly women with T1N0 oestrogen receptor (ER) positive $\mathrm{BC}$ undergoing $\mathrm{BCT}$ where the radiation fields include Level 1 axillary nodes. When a SLNB is performed in this subset of patients and 1 or 2 of the SLNs are negative, no further treatment of the axilla is required: this finding was established by the results of the ACOSOG*Z-0011 trial which has found global application despite deficiencies in accrual and follow-up. ${ }^{29}$

Other studies of the clinically negative axilla have found that long-term survival rates are similar irrespective of whether the axilla is dissected, irradiated or kept under surveillance..$^{30,31}$ However, it must be noted that in these studies as well as the meta-analysis reported by Sanghani, ${ }^{32}$ all patients received chemotherapy and targeted treatments so it is likely that differences in outcome related to the method of axillary management used may not be significant in the final analysis of survival.

SLNB is contraindicated in the initial management of inflammatory breast cancer as well as cancer with gross invasion of skin and muscle: obstruction and distortion of the lymphatics leads to high false negative rates. Neoadjuvant chemotherapy (NAC) is indicated to reduce the size of the primary malignancy and the ipsilateral lymph-node metastases with down staging of positive axillary lymph-nodes to pN0 being reported in $30-40 \%$ of patients, ${ }^{33}$ raising the possibility of SLNB in the clinically negative axilla post NAC. In the NSABP trial B-27,** 428 patients underwent a SLNB and ALND after NAC. Using radio colloid, SLNs were identified in $89 \%$ patients with a false negative rate of $8.4 \%$ and with overall accuracy for prediction of axillary nodal status after NAC of $95.6 \% .{ }^{34}$ Use of SLNB after NAC has the potential to spare $40 \%$ of women who become pN0 after NAC of any further treatment (ALND or radiation) to the axilla.

*American College of Surgeons Oncology Group Study Z0011.

**National Surgical Adjuvant Breast and Bowel Project trial B-27.

\section{Conclusion}

The adoption of BCT has offered improved breast cosmesis and function to women with early breast cancer as well as longer survival compared to MTX. The reasons for this improvement in survival are unproven but cannot be attributed to radiation alone: survival after mastectomy followed by chest wall irradiation is inferior to survival after BCT. ${ }^{6}$ The explanation may lie in the cytokine and growth factor accumulation in the increased area of surgical trauma following MTX which then impairs the patient's immune response. ${ }^{31}$ Lymphoedema is not an uncommon complication of ALND and is usually managed by complex physical therapies ${ }^{32}$ and compression garments. Despite the occasional success of lymphovascular anastomotic techniques, ${ }^{33}$ lymphoedema after ALND is incurable. SLNB has reduced the incidence of lymphoedema: when microscopic disease is identified in a SLN, adjuvant and targeted therapy have replaced ALND.

These observations support contemporary efforts to reduce surgical trauma to the breast and axilla in women with BC.

\section{REFERENCES}

1. Halsted W. The results of operations for the cure of cancer of the breast performed at the John Hopkins Hospital from June 1889 to January 1894. Ann Surg. 1894;20(5):497-555.

2. Cowan DH. Vera Peters and the Conservative management of early-stage breast cancer. Curr Oncol. 2010 April; 17(2):50-4.

3. The Curies Discover Radium. Accessed 21 Available from: https:www.wired.com/2009/12/1221 curries-discover radium

4. Keynes G. The radium treatment of carcinoma of the breast. $\mathrm{Br}$ Surg. 1931;19:425-43.

5. Keynes G. Conservative Treatment of Cancer of the Breast. BMJ. Oct 1937;643-7.

6. McWhirter R. The value of simple mastectomy and radiotherapy - the treatment of breast cancer. Br J Radiol. 1948;21:599-608.

7. Peters MV. Wedge resection and irradiation an effective treatment in early breast cancer. JAMA. 1967.

8. Fisher B, Anderson S, Bryant, et al. Twenty-year follow-up of a randomized trial comparing total mastectomy, lumpectomy and lumpectomy plus irradiation for the treatment of invasive breast cancer. N Engl J Med. 17 Oct 2002;347(6):1233-41. doi:10.1056/NEJ Moa 020989

9. Veronesi U, Salvadoni B, Luini A, et al. Breast conservation is a safe method in patients with small cancer of the breast. Longterm results of three randomized trials in 1973 patients. Eur J 


\section{SURGICAL LEADERSHIP PROGRAM}

A Certificate Program for Executive Skills Development and High-Impact Leadership

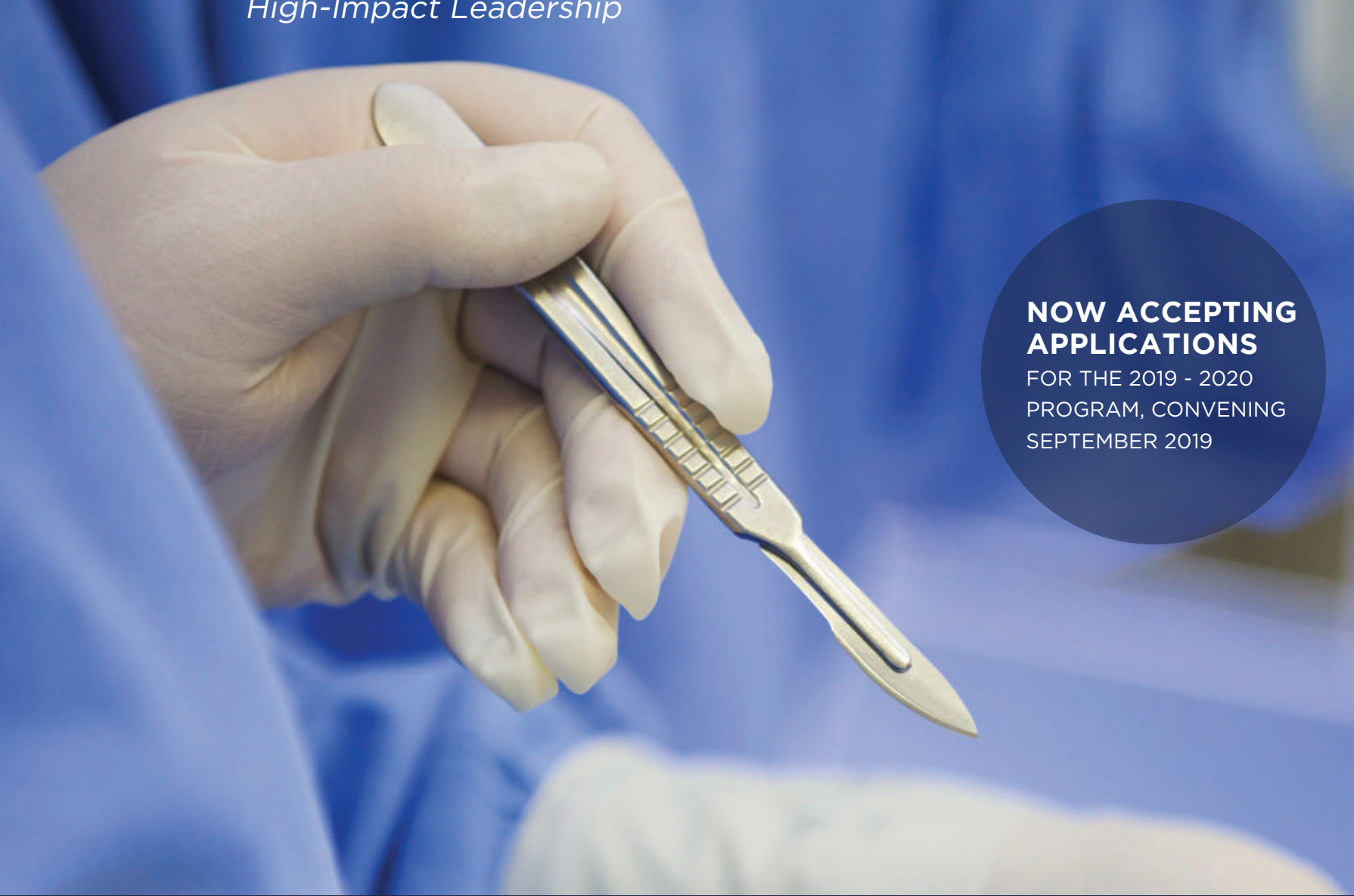

\section{Apply at HMS.Harvard.Edu/SurgicalLeadership}

Designed to accommodate the schedules of surgeons who currently hold or want to assume a leadership position:

Three four-day workshops at your choice of locations: Boston, London or Hong Kong

4 On-Demand Sessions 10 Live Online Webinars

Personalized Skills-Development Projects
The Surgical Leadership Program is the Harvard Medical School (HMS) postgraduate certificate program for surgeons seeking to succeed in leadership positions and acquire the skills needed to excel as heads of departments, divisions, projects and institutions at large. Participants learn directly from Harvard's leading faculty in key areas influencing surgical leadership, including communication, mentoring, process and quality improvement, change management, financial management and surgical research and technique. 
Cancer. 31 Sept 1995;10:1574-9.

10. NIH Consensus Development Conference Statement on the treatment of early-stage breast cancer. JAMA. 16 Jan 1991:265(3);391-5.

11. Van dongen JA, Voogd AC, Fentman IS, et al. Long-term results of a randomized trial comparing breast-conserving therapy with mastectomy: European Organization for Research and Treatment of Cancer. 10801 trial. J Natl Cancer Inst. 19 Jul 2000;92(14):1143-5.

12. Houssani N, Macaskill P, Marinovich ML, Morrow M. The association of surgical margins and local recurrence in women with early-stage invasive cancer treated with breast-conserving therapy: a meta-analysis. Ann Surg Oncol. Mar 2014;21(3):71730. doi:10.1245/5 10434-014-34080-5

13. Emmadi R, Wiley SL. Evaluation of resection margins in breast conservation therapy: the pathology perspective - past, present and future. Int J Surg Oncol. 2012:180-9. doi: $10,1155 / 2012 / 180259$

14. Gomez SL, Lichtensztayn D, Kurian AW, et al. Increasing mastectomy rates for early-stage breast cancer? Populationbased trends from California. Clin Breast Cancer. 2010;28(1):e155-e157 author reply e58. doi:10.1200/ jGO.2009.26.1032

15. Huang ES, Lichlenstain DX, Gomez SL, et al. Survival after lumpectomy and mastectomy for early stage invasive breast cancer: the effect of age and hormone receptor status. Cancer. Apr 2013;119(7):1402-11. doi:10.1002/cncr.27795

16. Agarwal S, Pappas L, Neumayer L, et al. Effect of Breast Conservation Therapy vs Mastectomy on DiseaseSpecific Survival for Early-stage Breast cancer. JAMA Surg. 2014:149(3):264-74. doi.10.1001/yamasurg. 2013. 304918.

17. Hafvind $\mathrm{S}$, Aden $\mathrm{A}$, Aas $\mathrm{T}$, et al. Women treated with breast conserving surgery do better than those with mastectomy independent of detection mode, prognostic and predictive tumour characteristics. Eur J Surg Oncol. Oct 2015[E pub 17 Jul 2015];41(10):1417-22. doi:10.1016/j.eyso.2015.07.002

18. Hartmann-Johnsen OJ, Kresen R, Schlichting E, Nygard JF. Survival is Better After Breast Conserving Therapy than Mastectomy for Early Stage Breast Cancer. A RegistryBased Follow-up Study of Norwegian Women Primary Operated Between 1998 and 2008. Ann. Surg. Oncol Nov 2015;22(12):3836-45. doi.1245/s 10434-015-4441-3. Ep20.

19. The American Joint Committee on Cancer. AJCC Staging Manual 7thy Edition. SB Edge 2010. Ann Surg Oncol. Jun 2010;17(6):1471-4. doi.10.1245/s 10434-010-0985421.

20. Tohe S, Simmons RL, Tsung A. Surgery for Cancer: A Trigger for Metastases. Cancer Res. Apr 2017;77(7):1548-52. doi:10.1158/0008-5472. CAN-16-1536

21. Da Costa ML, Redmond P, Bouchier-Hayes DJ. The effect of laparotomy and laparoscopy on the establishment of spontaneous tumour metastases. Surgery. Sep 1998;124(3):51625 .
22. Berguer R, Bravo N, Bowyer M, Egan C, et al. Major surgery suppresses maximal production of helper T-cell cytokines without potentiating the release of helper T-cell type 2 cytokines. Archives of Surgery. May 1999;134(5):540-4.

23. Morrow M, Strom EA, Bassett LW et al. Standard for breast conservation therapy in the management of invasive breast cancer. CA Cancer J Clin. Sept-Oct 2002;52:277-300. Available from: https//doi.org/10.3322/ca N Clin.52.5.277

24. Morton DL, Wen DR, Wong JH, et al. Technical details of intraoperative lymphatic mapping for early stage melanoma. Arch Surg. 1992:127(4):392-9.

25. Krag DN, Anderson SJ, Julian TB, et al. Technical outcomes of sentinel-lymph node resection and conventional axillarylymph-node dissection in patients with clinically node-negative breast cancer: results from the NSABPB-32 randomised phase III trial. Lancet Oncol. 8 Oct 2007; 8(10):881. doi:10.1016/S 1470-2045(07) 7028-4

26. Kissin MW, Thompson EM, Price AB, et al. The inadequacy of axillary sampling in breast cancer. Lancet. May 1982;319(8283):1210-2. Available from: https:/doi. org/10.1016/50140-6736(82) 92337-628.

27. Kim T, Guiliano AE, Lyman GH. Lymphatic mapping and sentinel lymph node biopsy in early-stage breast carcinoma: a meta-analysis cancer. 1 Jan 2006;106(1):4-16. doi:10.1002/ cncr.2156829.

28. Giuliano AE, McCali L, Beitsch $\mathrm{P}$, et al. Locoregional recurrence after sentinel lymph node dissection with or without axillary dissection in patients with sentinel lymph node metastases: the American College of Surgeons Oncology Group Z0011 randomized trial. Ann Surg. Sept 2010:252(3):426-33. doi:10.1097/SLA. Ob013e 3181f08f32

29. Donker M, Van Tienhoven G, Straver ME et al. Radiotherapy or surgery of the axilla after a positive sentinel node in breast cancer. (EORTC 10981-22023 AMAROS): a randomised, multicentre, open-label, phase 3 non-inferiority trial. Lancet Oncol. 2014;15(12):1303-10. doi:10.1016/514702045(14)70460-7

30. Louis-Sylvestre C, Clough K, Asselain B, et al. Axillary treatment in conservative management of operable breast cancer: dissection of radiotherapy? Results of a randomized study with 15 years of follow-up. J Clin Oncol. 1 Jan 2004;22:97-101. doi:10.1700/JCO.2004.12.108

31. Sanghani M, Balk EM, Cady B. Impact of axillary node dissection on breast cancer outcome in clinically node negative patients: a systematic review and meta-analysis. Cancer. 2009;115(8)1613-20. doi.10.1002/cncr.24174

32. Gianni L, Baselga J, Eiermann W, et al. First report of the European Cooperative trial in operable breast cancer (ECOTO): Effects of primary systemic therapy (PST) on local-regional disease. Proc Am Soc Clin Concol. 2002;21:34a (abstr 132)

33. Mamounas EP, Brown A, Anderson S, et al. Sentinel Node Biopsy After Neoadjuvant Chemotherapy in Breast Cancer: Results from National Surgical Adjuvant Breast and Bowel Project Protocol. B-27. J Clin Oncol. 20 Apr 2005:23(12):2694702. doi:10.1200/JCO.2005.05.188 